\title{
Asymptotic Dynamics of a Class of Third Order Rational Difference Equations
}

\author{
Sk. Sarif Hassan ${ }^{\circledR}$, Soma Mondal, Swagata Mandal ${ }^{\dagger} \&$ Chumki Sau ${ }^{\ddagger}$ \\ Department of Mathematics \\ Pingla Thana Mahavidyalaya \\ Maligram, Paschim Midnapur, 721140, India. \\ ${ }^{\circledR}$ Email:sarimif@gmail.com
}

April 6, 2020

\begin{abstract}
The asymptotic dynamics of the classes of rational difference equations (RDEs) of third order defined over the positive real-line as

$$
x_{n+1}=\frac{x_{n}}{a x_{n}+b x_{n-1}+c x_{n-2}}, x_{n+1}=\frac{x_{n-1}}{a x_{n}+b x_{n-1}+c x_{n-2}}, x_{n+1}=\frac{x_{n-2}}{a x_{n}+b x_{n-1}+c x_{n-2}}
$$

and

$$
x_{n+1}=\frac{a x_{n}+b x_{n-1}+c x_{n-2}}{x_{n}}, x_{n+1}=\frac{a x_{n}+b x_{n-1}+c x_{n-2}}{x_{n-1}}, x_{n+1}=\frac{a x_{n}+b x_{n-1}+c x_{n-2}}{x_{n-2}}
$$

is investigated computationally with theoretical discussions and examples. It is noted that all the parameters $a, b, c$ and the initial values $x_{-2}, x_{-1}$ and $x_{0}$ are all positive real numbers such that the denominator is always positive. Several periodic solutions with high periods of the RDEs as well as their inter-intra dynamical behaviours are studied.
\end{abstract}

Keywords: Rational difference equations, Local asymptotic stability, Periodic, Quasi-Periodic and Fractal-like trajectory.

Mathematics Subject Classification: 39A10 \& 39A11.

\section{Introduction}

Discrete dynamical systems is becoming very popular as it comes naturally in various biological systems including many other applied disciplines [1, 2]. In the past three decades, the theory of discrete dynamical systems and difference equations developed significantly. The theory of difference equations occupies a central position in applied sciences [3. Undoubtedly, the theory of difference equations will continue to play an important role in mathematics as a whole. The nonlinear difference equations of higher orders come quite naturally in modelling various systems we come across such as ecology, physiology, physics, engineering, economics, probability theory, genetics, psychology and resource management [4], [5], [6]. It is very interesting to investigate the behaviour of solutions of a higher-order rational difference equation and to discuss the local asymptotic stability of its equilibrium points [7, [8, [9]. Studying non-linear rational difference equations of higher orders is not though easy but very interesting with

\footnotetext{
*Summer Intern from the Department of Mathematics of Vidyasagar University at the Department of Mathematics, Pingla Thana Mahavidyalaya under Dr. Sk. Sarif Hassan.

†Summer Intern

$\ddagger$ Summer Intern
} 
enriched phenomena [10].

There are several textbooks available where the basic theory of rational difference equations of second and third order over the real lines are discussed [11, [12, [13. We are primarily concerned with the stability of the equilibrium points, the periodic character of the equation, and with convergence to periodic solutions and other dynamical behaviors if exists any [14].

There are 225 third order non-linear rational difference equations for which several thought-provoking open problems and conjectures on the boundedness character, the global stability, and the periodic behavior of their solutions are yet to close [15, [16, [17, [18, [19. In this present study, the rational difference equations (RDEs) we are interested to investigate are the following:

$$
\begin{aligned}
& x_{n+1}=\frac{x_{n}}{a x_{n}+b x_{n-1}+c x_{n-2}} \\
& x_{n+1}=\frac{x_{n-1}}{a x_{n}+b x_{n-1}+c x_{n-2}} \\
& x_{n+1}=\frac{x_{n-2}}{a x_{n}+b x_{n-1}+c x_{n-2}}
\end{aligned}
$$

These RDEs let us call Type-I and following RDEs, let us named as Type-II

$$
\begin{aligned}
& x_{n+1}=\frac{a x_{n}+b x_{n-1}+c x_{n-2}}{x_{n}} \\
& x_{n+1}=\frac{a x_{n}+b x_{n-1}+c x_{n-2}}{x_{n-1}} \\
& x_{n+1}=\frac{a x_{n}+b x_{n-1}+c x_{n-2}}{x_{n-2}}
\end{aligned}
$$

It is mentioned that all the parameters $a, b, c$ and the initial values $x_{-2}, x_{-1}$ and $x_{0}$ are all positive real numbers such that the denominator is always positive. It is noted that the rational difference equations 1, 2 and 3 of Type-II are same as 4 , 5 and 6 respectively.

\section{Local Asymptotic Stability of the Equilibrium points of RDEs of Type-I and Type-II}

The only positive equilibrium point of the equations 1, 2, 3 of Type-I and equations 4, 5, 6 of Type-II are $\frac{1}{a+b+c}$ and $a+b+c$ respectively. In the following two sub-sections we would see the local asymptotic stability of the equilibriums of the two types of RDEs.

Before we proceed into the subsections, we wish to present two important theorems which would be used [2].

Theorem 2.1. Assume that $a_{2}, a_{1}$, and $a_{0}$ are real numbers. Then a necessary and sufficient condition for all roots of the equation

$$
\lambda^{3}+a_{2} \lambda^{2}+a_{1} \lambda+a_{0}=0
$$

to lie inside the unit disk is

$\left|a_{2}+a_{0}\right|<1+a_{1},\left|a_{2}-3 a_{0}\right|<3-a_{1}$ and $a_{0}^{2}+a_{1}-a_{0} a_{2}<1$ 
Theorem 2.2. Assume that $a_{0}, a_{1}, a_{2}$ are real numbers such that

$$
\left|a_{0}\right|+\left|a_{1}\right|+\left|a_{2}\right|<1
$$

then all roots of equation $\lambda^{3}+a_{2} \lambda^{2}+a_{1} \lambda+a_{0}=0$ lie inside the unit disk.

\subsection{Local Asymptotic Stability of the Type-I RDEs}

The non-zero positive equilibrium point $(\bar{x})$ of 1,2 and 3 is the solutions of the equation

$$
\bar{x}=\frac{\bar{x}}{a \bar{x}+b \bar{x}+c \bar{x}}
$$

It turns out that $\frac{1}{a+b+c}$ is the only equilibrium point of the Type-I RDEs. The linearized equation of the rational difference equation 1 with respect to the equilibrium point $\frac{1}{a+b+c}$ is

$$
y_{n+1}=\frac{b+c}{a+b+c} y_{n}-\frac{b}{a+b+c} y_{n-1}-\frac{c}{a+b+c} y_{n-2}
$$

with associated characteristic equation

$$
\lambda^{3}-\frac{b+c}{a+b+c} \lambda^{2}+\frac{b}{a+b+c} \lambda+\frac{c}{a+b+c}=0
$$

Theorem 2.3. The necessary and sufficient for local asymptotic stability of the equilibrium point $\frac{1}{a+b+c}$ of the 1 of Type-I is

$$
a+\sqrt{a(2 a-b}>b+c
$$

Proof. By setting the conditions as stated in the Theorem 2.1 into the characteristic equation 18 and by simplification once can easily derive the necessary and sufficient condition for the local asymptotic stability of the equilibrium point $\frac{1}{a+b+c}$ of the 1 of Type-I is

$$
a+\sqrt{a(2 a-b}>b+c
$$

The linearized equation of the rational difference equation 2 with respect to the equilibrium point $\frac{1}{a+b+c}$ is

$$
y_{n+1}=-\frac{a}{a+b+c} y_{n}+\frac{a+c}{a+b+c} y_{n-1}-\frac{c}{a+b+c} y_{n-2}
$$

with associated characteristic equation

$$
\lambda^{3}+\frac{a}{a+b+c} \lambda^{2}-\frac{a+c}{a+b+c} \lambda+\frac{c}{a+b+c}=0
$$

Theorem 2.4. The necessary and sufficient for local asymptotic stability of the equilibrium point $\frac{1}{a+b+c}$ of the 2 of Type-I is

$$
a+c<b
$$

The linearized equation of the rational difference equation 2 with respect to the equilibrium point $\frac{1}{a+b+c}$ is

$$
y_{n+1}=-\frac{a}{a+b+c} y_{n}-\frac{b}{a+b+c} y_{n-1}+\frac{a+b}{a+b+c} y_{n-2}
$$

with associated characteristic equation

$$
\lambda^{3}+\frac{a}{a+b+c} \lambda^{2}+\frac{b}{a+b+c} \lambda-\frac{a+b}{a+b+c}=0
$$


Theorem 2.5. The necessary and sufficient for local asymptotic stability of the equilibrium point $\frac{1}{a+b+c}$ of the 3 of Type-I is

$$
2 a+b+2 c>\sqrt{8 a^{2}+4 a b+b^{2}}
$$

We have found computationally 10000 points $(a, b, c)$ of the RDEs of the Type-I such that each of the Theorems 2.3, 2.4 and 2.5 hold good. The points $(a, b, c)$ are plotted in three dimension as shown in Fig.1.
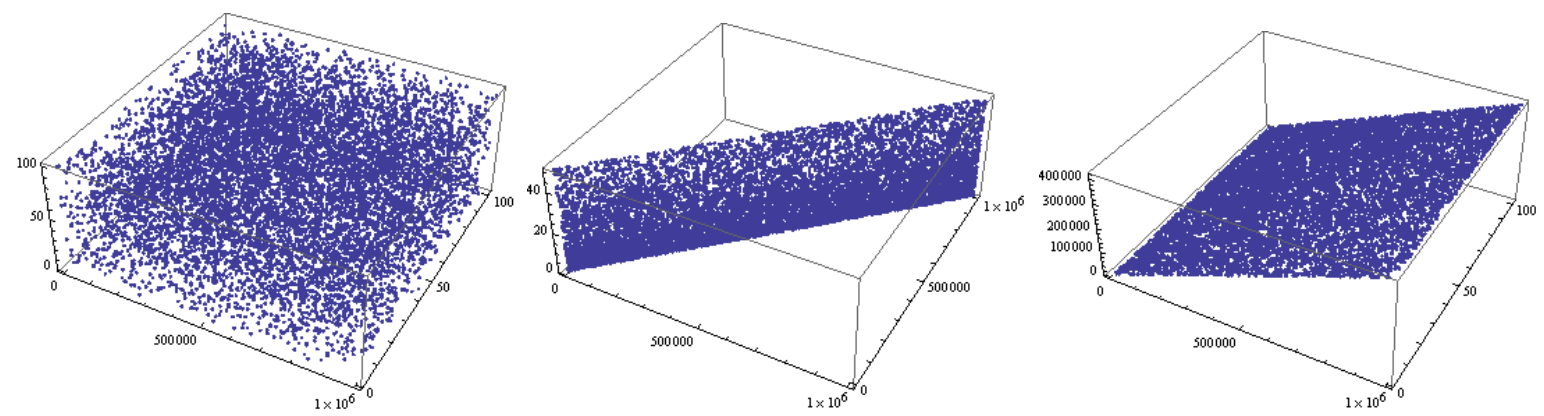

Figure 1: Space of parameters $a, b$ and $c$ such that the equilibrium of the RDEs of Type-I are local asymptotically stable.

Example 2.1. Consider $a=0.1998, b=0.6073$ and $c=0.5430$, then for ten different initial values taken from the neighbourhood of the equilibrium point 0.7406, the trajectories for the RDE 1 are convergent and converge to 0.7406 as shown in Fig 2.

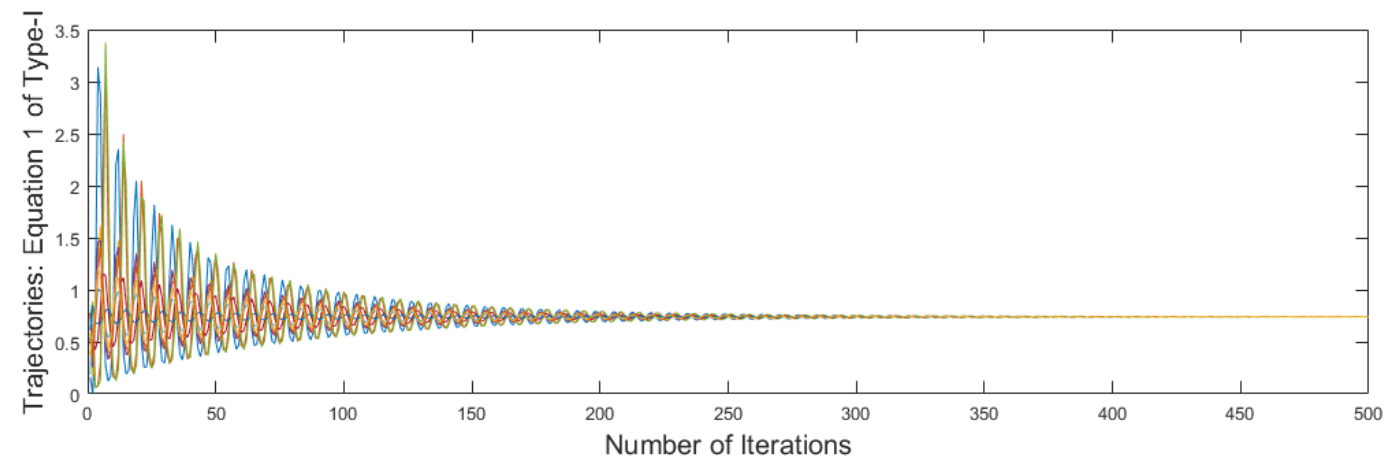

Figure 2: Local Asymptotic Stability of the equilibrium point of the RDE 1 of the Type-I

Example 2.2. Consider $a=0.0398, b=0.7092$ and $c=0.6413$, then for ten different initial values taken from the neighbourhood of the equilibrium point 0.7192, the trajectories for the RDE 2 are convergent and converge to 0.7192 as shown in Fig 3. 


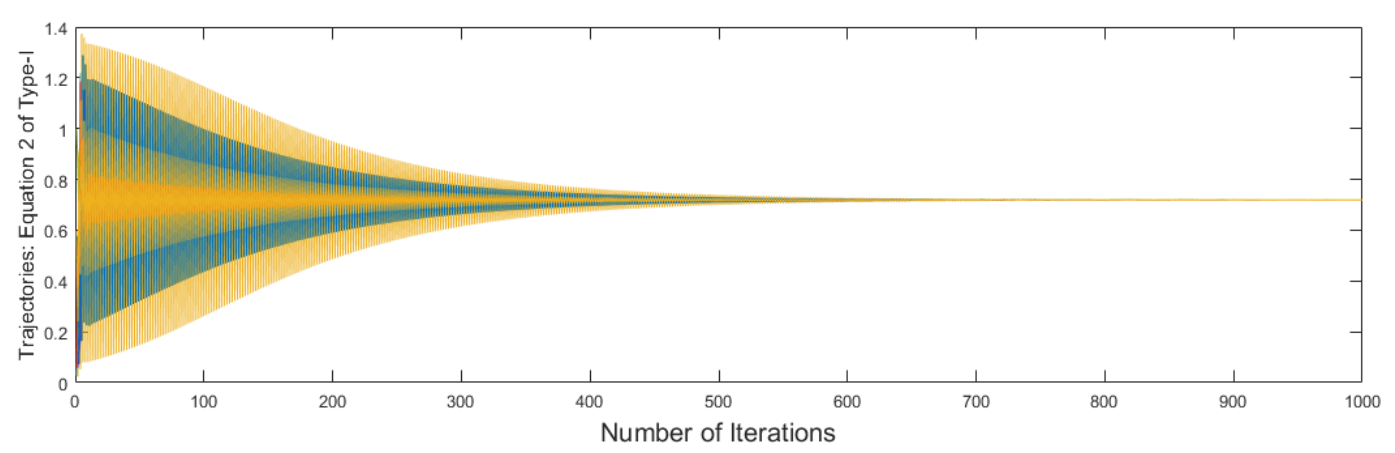

Figure 3: Local Asymptotic Stability of the equilibrium point for the RDE 2 of the Type-I

Example 2.3. Consider $a=0.3360, b=0.1448$ and $c=0.2449$, then for ten different initial values taken from the neighbourhood of the equilibrium point 1.378, the trajectories for the RDE 3 are convergent and converge to 1.378 as shown in Fig 4).

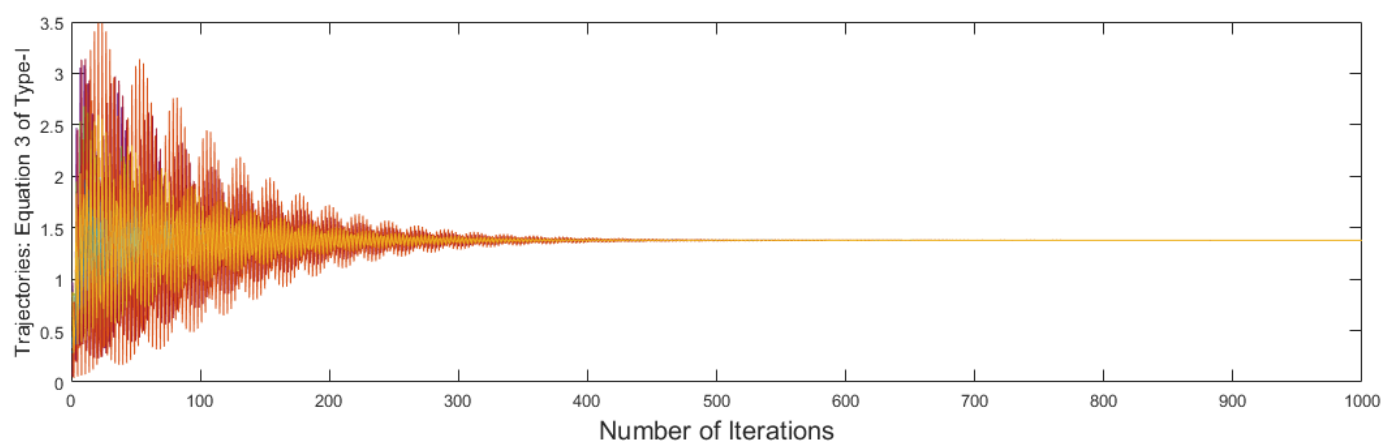

Figure 4: Local Asymptotic Stability of the equilibrium point for the RDE 3 of the Type-I

\subsection{Local Asymptotic Stability of the Type-II RDEs}

The non-zero positive equilibrium point $(\bar{x})$ of 4,5 and 6 is the solutions of the equation

$$
\bar{x}=\frac{a \bar{x}+b \bar{x}+c \bar{x}}{\bar{x}}
$$

It turns out that $a+b+c$ is the only equilibrium point of the Type-II RDEs. The linearized equation of the rational difference equation 4 with respect to the equilibrium point $a+b+c$ is

$$
y_{n+1}=\left(\frac{a}{a+b+c}-1\right) y_{n}+\frac{b}{a+b+c} y_{n-1}+\frac{c}{a+b+c} y_{n-2}
$$

with associated characteristic equation

$$
\lambda^{3}+\left(1-\frac{a}{a+b+c}\right) \lambda^{2}-\frac{b}{a+b+c} \lambda-\frac{c}{a+b+c}=0
$$

Theorem 2.6. The necessary and sufficient for local asymptotic stability of the equilibrium point $a+b+c$ of the 4 of Type-II is

$$
\sqrt{(a+b)(2 a+b)}+a, a+c>b, a<b
$$


The linearized equation of the rational difference equation 5 with respect to the equilibrium point $a+b+c$ is

$$
y_{n+1}=\frac{a}{a+b+c} y_{n}+\left(\frac{b}{a+b+c}-1\right) y_{n-1}+\frac{c}{a+b+c} y_{n-2}
$$

with associated characteristic equation

$$
\lambda^{3}+\frac{a}{a+b+c} \lambda^{2}+\left(1-\frac{b}{a+b+c}\right) \lambda+\frac{c}{a+b+c}=0
$$

Theorem 2.7. The necessary and sufficient for local asymptotic stability of the equilibrium point $a+b+c$ of the 5 of Type-II is

$$
a+2 b>\sqrt{5}
$$

The linearized equation of the rational difference equation 6 with respect to the equilibrium point $a+b+c$ is

$$
y_{n+1}=\frac{a}{a+b+c} y_{n}+\frac{b}{a+b+c} y_{n-1}-\frac{a+b}{a+b+c} y_{n-2}
$$

with associated characteristic equation

$$
\lambda^{3}-\frac{a}{a+b+c} \lambda^{2}-\frac{b}{a+b+c} \lambda+\frac{a+b}{a+b+c}=0
$$

Theorem 2.8. The necessary and sufficient for local asymptotic stability of the equilibrium point $a+b+c$ of the 6 of Type-II is

$$
\sqrt{3} a \geq a+2 b, 2 a+3 b+2 c>\sqrt{8 a^{2}+4 a b+b^{2}}
$$

We have found computationally 10000 points $(a, b, c)$ for all the RDEs of Type-II such that each of the Theorems 2.3, 2.4 and 2.5 hold good. The points $(a, b, c)$ are plotted in three dimension as shown in Fig.5.
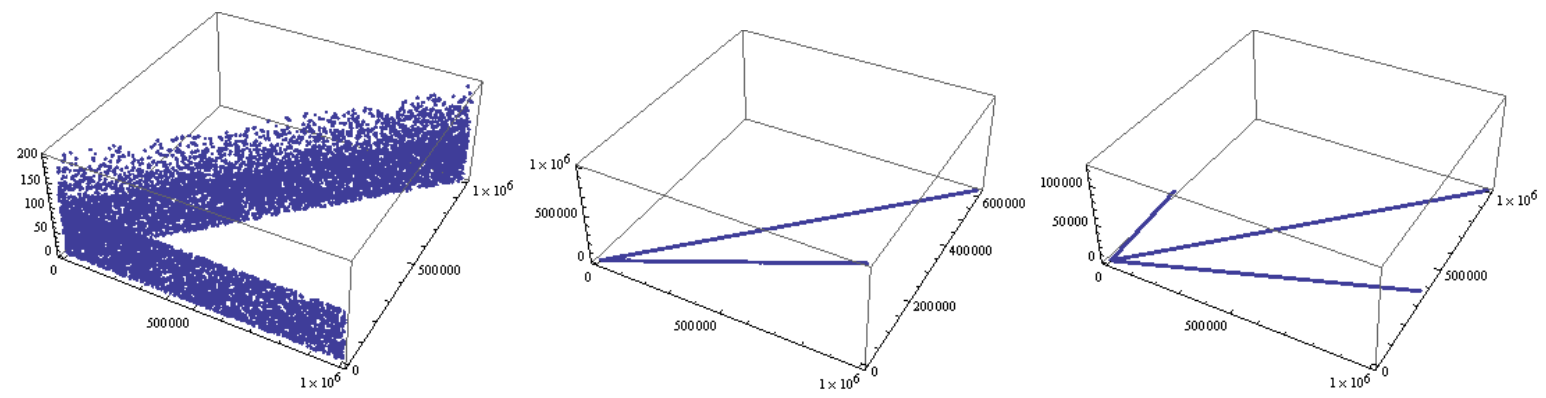

Figure 5: Space of parameters $a, b$ and $c$ such that the equilibrium of the RDEs of Type-II are local asymptotically stable.

Example 2.4. Consider $a=0.4984, b=0.8090$ and $c=0.3565$, then for ten different initial values taken from the neighbourhood of the equilibrium point 1.6639, the trajectories for the RDE 4 are convergent and converge to 1.6639 as shown in Fig 6. 


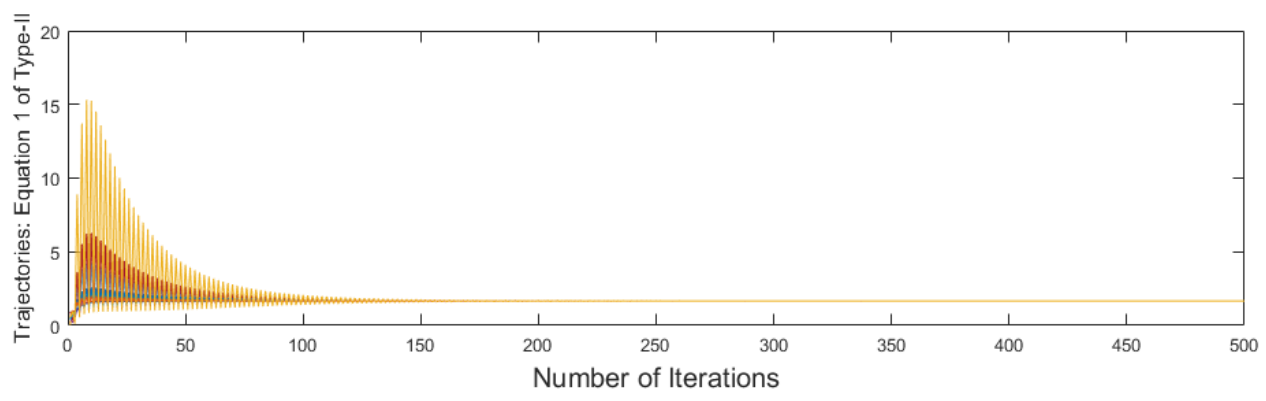

Figure 6: Local Asymptotic Stability of the equilibrium point of the RDE 4 of the Type-II

Example 2.5. Consider $a=0.8257, b=0.4445$ and $c=0.9821$, then for ten different initial values taken from the neighbourhood of the equilibrium point 2.2523, the trajectories for the RDE 5 are convergent and converge to 2.2523 as shown in Fig 7.

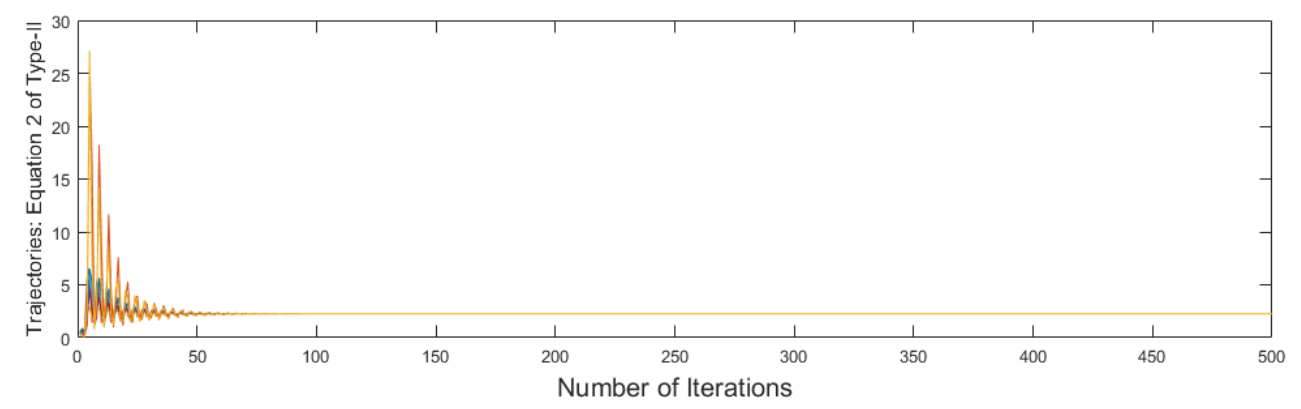

Figure 7: Local Asymptotic Stability of the equilibrium point for the RDE 5 of the Type-II

Example 2.6. Consider $a=0.2954, b=0.3661$ and $c=0.3490$, then for ten different initial values taken from the neighbourhood of the equilibrium point 1.0106, the trajectories for the RDE 6 are convergent and converge to 1.0106 as shown in Fig 8.

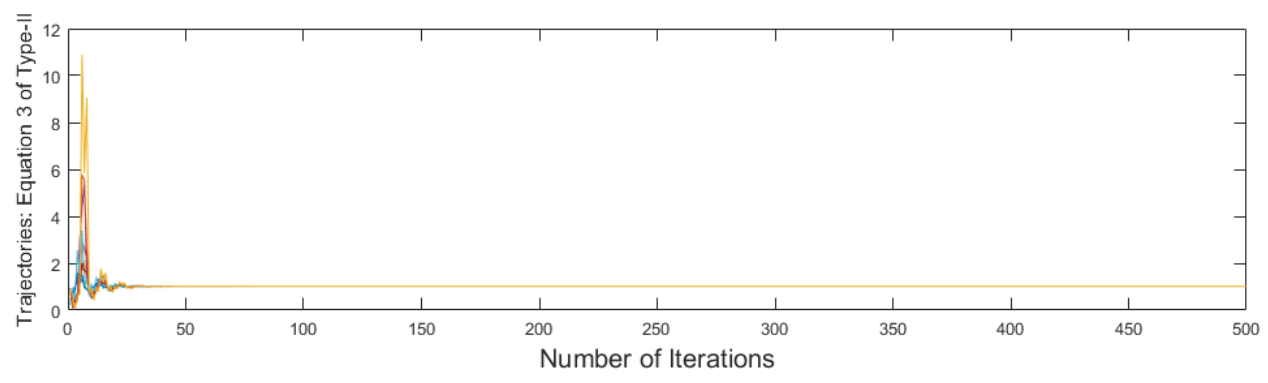

Figure 8: Local Asymptotic Stability of the equilibrium point for the RDE 6 of the Type-II

\subsection{Local Asymptotic Stability Criteria for all the RDEs of Type-I, Type-II}

Here we wish to derive the necessary and sufficient conditions for local asymptotic stability about the equilibrium point $\frac{1}{a+b+c}$ of all the three RDEs 1, 2 and 3 of Type-I, simultaneously. Same we shall do 
for the RDEs for Type-II.

Theorem 2.9. The equilibrium point $\frac{1}{a+b+c}$ of all the three RDEs 1, 2 and 3 of Type-I is locally asymptotically stable simultaneously if and only if

$$
\frac{8 b}{7+\sqrt{17}}<a<\frac{4 b}{1+\sqrt{17}}, \frac{1}{2} \sqrt{8 a^{2}+4 a b+b^{2}}+\frac{1}{2}(-2 a-b)<c<b-a
$$

Example 2.7. Here we list a set of parameters $a, b$ and $c$ for which the Theorem 2.9 holds good.

$$
\begin{gathered}
\left\{a \rightarrow 184, b \rightarrow \frac{1049}{4}, c \rightarrow \frac{163}{3}\right\},\left\{a \rightarrow 131, b \rightarrow \frac{701}{4}, c \rightarrow \frac{549}{13}\right\},\left\{a \rightarrow 669, b \rightarrow \frac{1783}{2}, c \rightarrow 190\right\} \\
\left\{a \rightarrow 180, b \rightarrow \frac{1045}{4}, c \rightarrow \frac{1453}{28}\right\},\left\{a \rightarrow 87, b \rightarrow 116, c \rightarrow \frac{601}{21}\right\},\left\{a \rightarrow 598, b \rightarrow \frac{1533}{2}, c \rightarrow \frac{26696}{159}\right\}
\end{gathered}
$$

Theorem 2.10. The equilibrium point $a+b+c$ of all the three RDEs 4, 5 and 6 of Type-II is locally asymptotically stable simultaneously if and only if

$$
\sqrt{(a+b)(2 a+b)}+a>c, a+c>b, b<a
$$

Example 2.8. Consider $a=0.3685, b=0.6256, c=0.7802$, then all the three $R D E s 4,5$ and 6 are convergent simultaneously and converge to the equilibrium 1.1774 as shown in the following Fig.9.
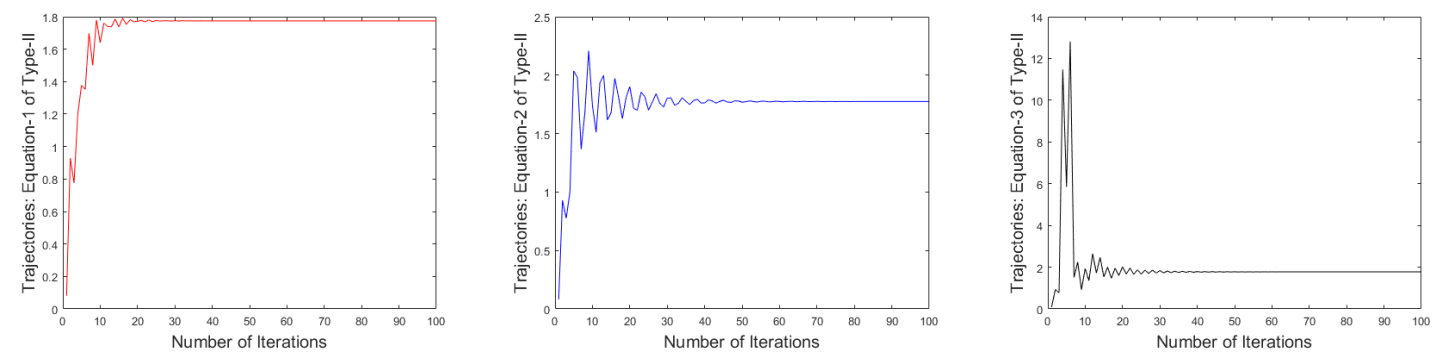

Figure 9: Local asymptotic stability of the equilibrium for all the three RDEs simultaneously.

Now we shall find the necessary and sufficient conditions for local asymptotic stability of the equilibria $\frac{1}{a+b+c}$ for REDs of Type-I and RDEs of Type-II simultaneously.

Theorem 2.11. The necessary and sufficient conditions for local asymptotic stability of the equilibria $\frac{1}{a+b+c}$ for 1 of Type-I and $a+b+c$ of 4 of Type-II simultaneously is

$$
\sqrt{a(2 a-b)}+a>b+c, b \leq a
$$

Example 2.9. Consider $a=0.5470, b=0.2963, c=0.7447$, then the equilibria 0.6297 for 1 of Type-I and 1.5880 for 4 of Type-II are convergent simultaneously as shown in the following Fig.10. 

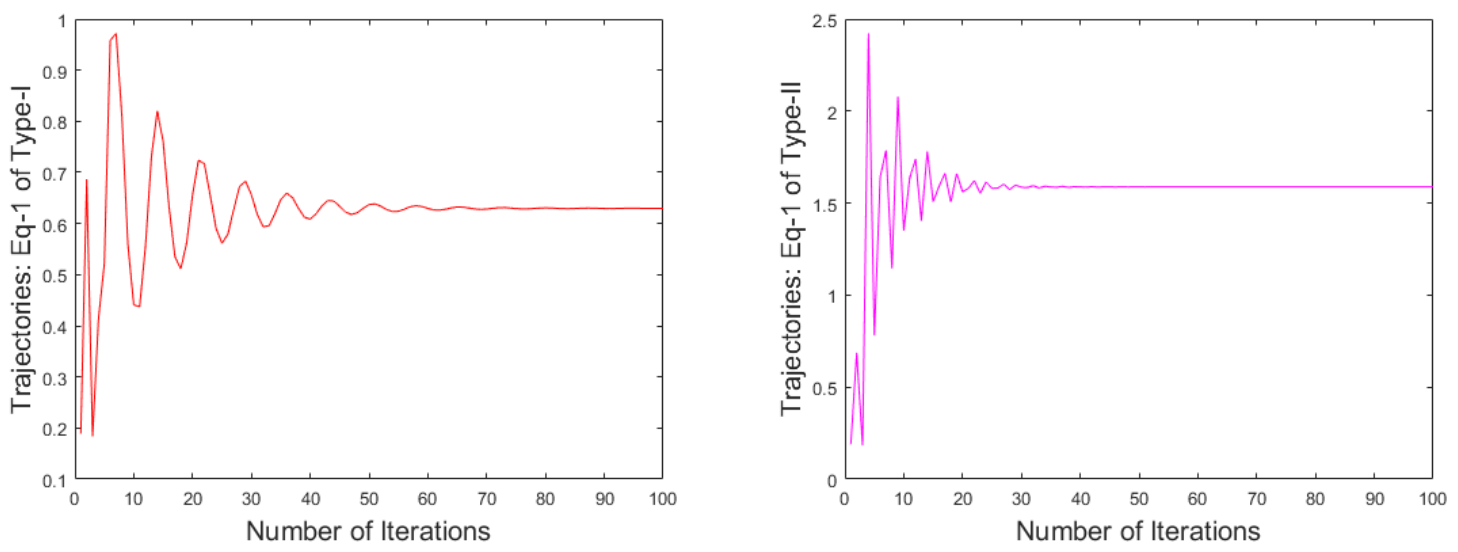

Figure 10: Local asymptotic stability of the equilibria for 1 of Type-I and for 4 of Type-II, simultaneously.

Theorem 2.12. The necessary and sufficient conditions for local asymptotic stability of the equilibria $\frac{1}{a+b+c}$ for 2 of Type-I and $a+b+c$ of 5 of Type-II simultaneously is

$$
a+c<b, b>a
$$

Example 2.10. Consider $a=0.2920, b=0.4317, c=0.0155$, then the equilibria 1.3530 for 2 of Type-I and 0.7391 for 5 of Type-II are convergent simultaneously as shown in the following Fig.11.
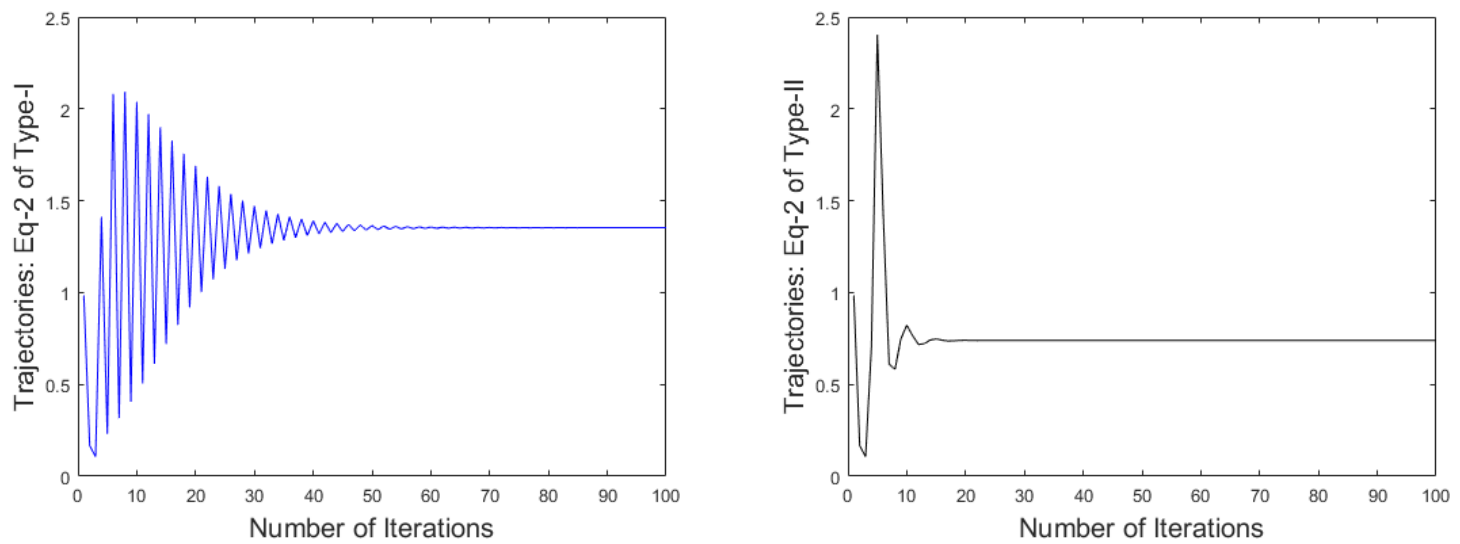

Figure 11: Local asymptotic stability of the equilibria for 2 of Type-I and for 5 of Type-II, simultaneously.

Theorem 2.13. The necessary and sufficient conditions for local asymptotic stability of the equilibria $\frac{1}{a+b+c}$ for 3 of Type-I and $a+b+c$ of 6 of Type-II simultaneously is

$$
\sqrt{3} a \geq a+2 b, 2 a+b+2 c>\sqrt{8 a^{2}+4 a b+b^{2}}
$$

Example 2.11. Consider $a=0.4401, b=0.5271, c=0.4574$, then the equilibria 0.7019 for 3 of Type-I and 1.4247 for 6 of Type-II are convergent simultaneously as shown in the following Fig.12. 

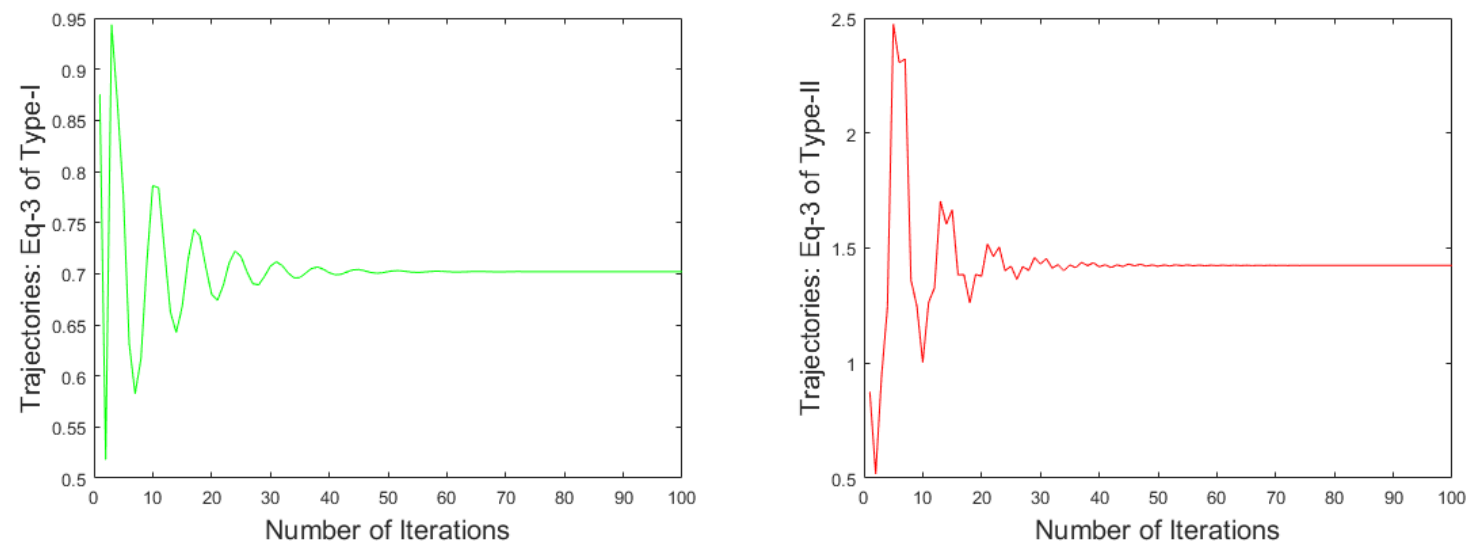

Figure 12: Local asymptotic stability of the equilibria for 3 of Type-I and for 6 of Type-II, simultaneously.

\subsection{Sufficient Condition for Local Asymptotic Stability of RDEs}

Here we shall derive the sufficient conditions for local asymptotic stability of the equilibria $\frac{1}{a+b+c}$ for RDEs of Type-I and $a+b+c$ for RDEs of Type-II.

Theorem 2.14. The equilibrium $\frac{1}{a+b+c}$ is locally asymptotically stable for the RDE 1 if $b+c<a$.

Theorem 2.15. The equilibrium $\frac{1}{a+b+c}$ is locally asymptotically stable for the RDE 2 if $a+c<b$.

Theorem 2.16. The equilibrium $\frac{1}{a+b+c}$ is locally asymptotically stable for the RDE 3 if $a+b<c$.

Theorem 2.17. The equilibrium $a+b+c$ is locally asymptotically stable for the $R D E 4$ if $b+c<a$.

Theorem 2.18. The equilibrium $a+b+c$ is locally asymptotically stable for the $R D E 5$ if $a+c<b$.

Theorem 2.19. The equilibrium $a+b+c$ is locally asymptotically stable for the $R D E 6$ if $a+b<c$.

Remark 2.1. It is obtained that the sufficient conditions for RDE 1 of Type-I and RDE 4 of Type-II are identical. Same is true for the RDE 2 of Type-I and RDE 5 of Type-II as well as for RDE 3 of Type-I and RDE 6 of Type-II.

Remark 2.2. It is worth mentioning that the Theorems 2.11,2.12 and 2.13 do not hold simultaneously and consequently the Theorems 2.14,2.15 and 2.16 do not hold good.

\section{Periodic Solutions of the RDEs}

The necessary condition for the existence of prime period-two solutions of the general third order rational equation

$$
x_{n+1}=\frac{\alpha+\beta x_{n}+\gamma x_{n-1}+\delta x_{n-2}}{A+B x_{n}+C x_{n-1}+D x_{n-2}}
$$

with nonnegative parameters $\alpha, \beta, \gamma, A, B, C, D$ and with arbitrary non-negative initial conditions $x_{-2}, x_{-1}, x_{0}$ such that the denominator is always positive is

$$
C(\phi+\psi)=\gamma-\beta-\delta-A
$$


where $\phi$ and $\psi$ are prime period two solutions of the general third order RDE [11],[13], 20], 21].

Let us discover the existence of the prime period two solutions of the RDEs of Type-I and Type-II before we actually proceed to discover the prime period two solutions of the RDEs. Now by comparing the RDEs of Type-I and Type-II with the general third order RDE, we have the parameters as the following shown in the Table-1:

\begin{tabular}{|c|l|}
\hline RDEs of Type-I and Type-II & Parameters \\
\hline$x_{n+1}=\frac{x_{n}}{a x_{n}+b x_{n-1}+c x_{n-2}}$ & $\alpha=0, \beta=1, \gamma=0, \delta=0, \mathrm{~A}=0, \mathrm{~B}=\mathrm{a}, \mathrm{C}=\mathrm{b}, \mathrm{D}=\mathrm{c}$ \\
\hline$x_{n+1}=\frac{x_{n-1}}{a x_{n}+b x_{n-1}+c x_{n-2}}$ & $\alpha=0, \beta=0, \gamma=1, \delta=0, \mathrm{~A}=0, \mathrm{~B}=\mathrm{a}, \mathrm{C}=\mathrm{b}, \mathrm{D}=\mathrm{c}$ \\
\hline$x_{n+1}=\frac{x_{n-2}}{a x_{n}+b x_{n-1}+c x_{n-2}}$ & $\alpha=0, \beta=0, \gamma=0, \delta=1, \mathrm{~A}=0, \mathrm{~B}=\mathrm{a}, \mathrm{C}=\mathrm{b}, \mathrm{D}=\mathrm{c}$ \\
\hline$x_{n+1}=\frac{a x_{n}+b x_{n-1}+c x_{n-2}}{x_{n}}$ & $\alpha=0, \beta=a, \gamma=b, \delta=c, \mathrm{~A}=0, \mathrm{~B}=1, \mathrm{C}=0, \mathrm{D}=0$ \\
\hline$x_{n+1}=\frac{a x_{n}+b x_{n-1}+c x_{n-2}}{x_{n-1}}$ & $\alpha=0, \beta=a, \gamma=b, \delta=c, \mathrm{~A}=0, \mathrm{~B}=0, \mathrm{C}=1, \mathrm{D}=0$ \\
\hline$x_{n+1}=\frac{a x_{n}+b x_{n-1}+c x_{n-2}}{x_{n-2}}$ & $\alpha=0, \beta=a, \gamma=b, \delta=c, \mathrm{~A}=0, \mathrm{~B}=0, \mathrm{C}=0, \mathrm{D}=1$ \\
\hline
\end{tabular}

Table 1: Relationship of Parameters of the RDEs with the general third order RDE.

For the RDEs 1 and 3 of Type-I from the parameters given in the Table 1, the equation 19 becomes $b(\phi+\psi)=-1$ which is impossible to occur as $b, \phi$ and $\psi$ are all positive real numbers. Hence the RDEs 1 and 3 of Type-I do not possess any prime period two solution.

The equation 19 for the RDE 2 of Type-I becomes $b(\phi+\psi)=1$, which is absolutely possible for certain choices of the parameters and prime period two solutions $\phi$ and $\psi$. Hence the prime period two solution exists for the RDE 2 of Type-I. One of the prime period two solutions is essentially turns out to be $0, \frac{1}{b}, 0, \frac{1}{b}, 0, \frac{1}{b} \ldots \ldots$

The equation 19 for the RDEs 4 and 6 of Type-II becomes $b=a+c$, which is certainly possible for certain choices of the parameters and prime period two solutions $\phi$ and $\psi$. Hence the prime period two solution exists for the RDEs 4 and 6 of Type-II.

The equation 19 for the RDE 5 of Type-II becomes $(\phi+\psi)=b-a-c$. This is possible only if $b>a+c$. Hence the prime period two solution exists for the RDE 5 of Type-I whenever $b>a+c$.

\subsection{Periodic Solutions of the RDEs of Type-I}

The RDE 2 has prime period two solutions as confirmed theoretically and computationally. Let $\phi, \psi, \phi, \psi, \ldots, \ldots$ is a prime period two solution of the RDE 2 . Then it must satisfy the identity $b(\phi+\psi)=1$.

if $\phi=0$ then by the identity we get $\psi=\frac{1}{b}$. Hence $0, \frac{1}{b}, 0, \frac{1}{b}, \ldots$ turns out to be a prime period two solution of the RDE 2 .

Example 3.1. Here We list a set of parameters such that the RDE 2 of Type-I would possess prime period two solutions $0, \frac{1}{b}, 0, \frac{1}{b}, \ldots \ldots$. The list is given in the Table 2 and the corresponding prime period two solutions plot are shown in the Fig. 13. 


\begin{tabular}{|c|l|l|}
\hline Parameters & $\frac{1}{b}$ & Remarks \\
\hline $\begin{array}{l}\mathrm{a}=0.7851, \\
\mathrm{~b}=0.0741, \\
\mathrm{c}=0.3939\end{array}$ & 13.4953 & $\phi=0$ and $\psi=13.4953$ as shown in Fig. (Row-1, Col-1) \\
\hline $\begin{array}{l}\mathrm{a}=0.1892, \\
\mathrm{~b}=0.1425, \\
\mathrm{c}=0.2681\end{array}$ & 7.0175 & $\phi=0$ and $\psi=7.0175$ as shown in Fig. (Row-1, Col-2) \\
\hline $\begin{array}{l}\mathrm{a}=0.9011, \\
\mathrm{~b}=0.9394, \\
\mathrm{c}=0.2212\end{array}$ & 1.0645 & $\phi=0$ and $\psi=1.0645$ as shown in Fig. (Row-1, Col-3) \\
\hline $\begin{array}{l}\mathrm{a}=0.2649, \\
\mathrm{~b}=0.0684, \\
\mathrm{c}=0.4363\end{array}$ & 14.6199 & $\phi=0$ and $\psi=14.6199$ as shown in Fig. (Row-2, Col-1) \\
\hline $\begin{array}{l}\mathrm{a}=0.4306, \\
\mathrm{~b}=0.9616, \\
\mathrm{c}=0.7624\end{array}$ & 1.0399 & $\phi=0$ and $\psi=1.0399$ as shown in Fig. (Row-2, Col-2) \\
\hline $\begin{array}{l}\mathrm{a}=0.6451, \\
\mathrm{~b}=0.5523, \\
\mathrm{c}=0.2181\end{array}$ & 1.8106 & $\phi=0$ and $\psi=1.8106$ as shown in Fig. (Row-2, Col-3) \\
\hline
\end{tabular}

Table 2: Parameters and corresponding prime period two solutions of the RDE 1.
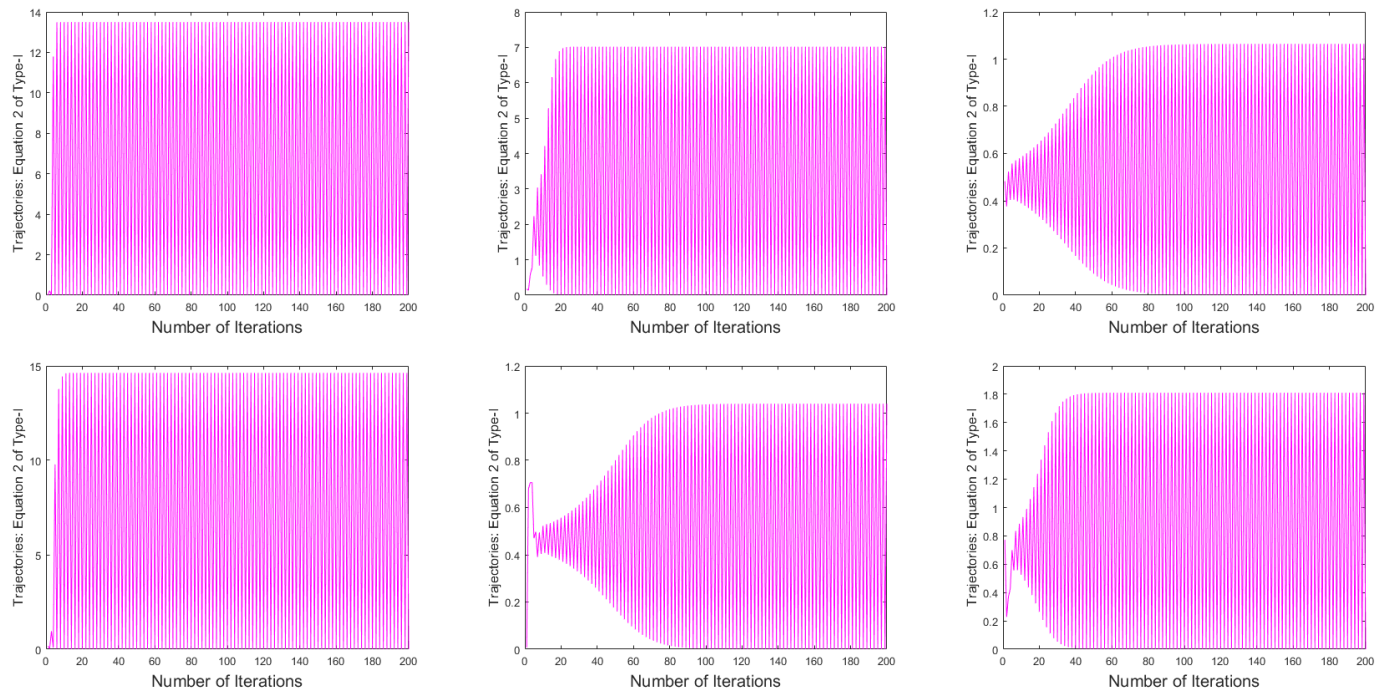

Figure 13: Prime period two solutions $0, \frac{1}{b}, 0, \frac{1}{b}, \ldots \ldots$ for different set of parameters

When $b=1$, then the identity results to a prime period two solutions $\phi, 1-\phi, \phi, 1-\phi, \ldots \ldots$ Here we present a couple of examples such prime period two solutions of the RDE 2 of Type-I.

Example 3.2. Here We list a set of parameters such that the RDE 2 of Type-I would possess prime period two solutions $\phi, 1-\phi, \phi, 1-\phi, \ldots \ldots$. The list is given in the Table 3 and the corresponding prime period two solutions plot are shown in the Fig. 14. 


\begin{tabular}{|c|l|l|}
\hline Parameters & $\phi, \psi=1-\phi$ & Remarks \\
\hline $\begin{array}{c}\mathrm{a}=0.4301, \mathrm{~b}=1, \\
\mathrm{c}=0.5722\end{array}$ & $\phi=0.4921, \psi=0.5067$ & as shown in Fig. 14 (left) \\
\hline $\begin{array}{c}\mathrm{a}=0.3367, \mathrm{~b}=1, \\
\mathrm{c}=0.6624\end{array}$ & $\phi=0.6927, \psi=0.3077$ & as shown in Fig. 14 (middle) \\
\hline $\begin{array}{c}\mathrm{a}=0.9345, \mathrm{~b}=1, \\
\mathrm{c}=0.1079\end{array}$ & $\phi=0.5272, \psi=0.4517$ & as shown in Fig. 14 (right) \\
\hline
\end{tabular}

Table 3: Parameters with corresponding prime period two solutions when $b=1$ of the RDE 2
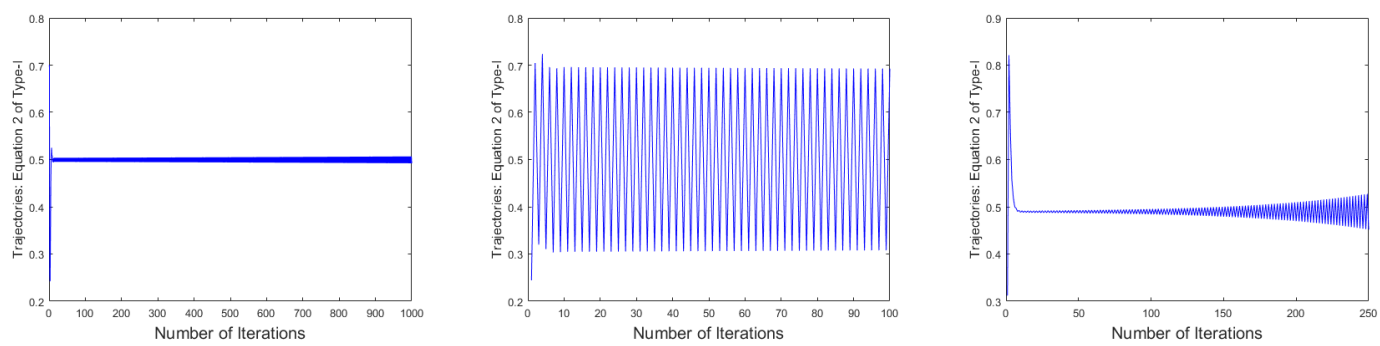

Figure 14: Prime period two solutions other than $\{0,1,0,1, \ldots\}$ of the RDE 2 of Type-I.

Now we shall try to explore any other higher periodic solutions (if exists any) of the RDEs of Type-I. Here we exhibit some quasi-periodic (near-periodic) with high quasi-period (near-period) solutions of the RDE 1 of Type-I as shown in the following two examples 3.3 and 3.4.

Example 3.3. Consider $a=0.1525, b=0.0196$ and $c=0.4352$ with initial values taken from the $\epsilon$ - $n b d$ of the zero, the trajectory possesses to a very high quasi periodic solution as shown in Fig.15.

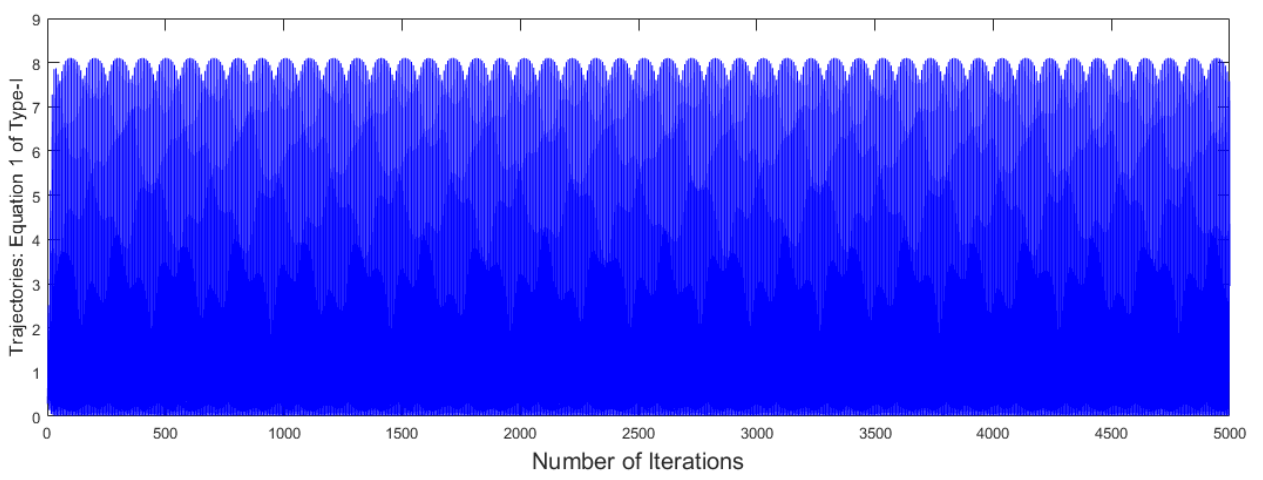

Figure 15: Quasi-periodic solution of the RDE 1.

Example 3.4. Consider $a=0.1161, b=0.0577$ and $c=0.9798$ with initial values taken from the $\epsilon$ - $n b d$ of the zero, the trajectory possesses to a very high quasi periodic solution as shown in Fig.16. 


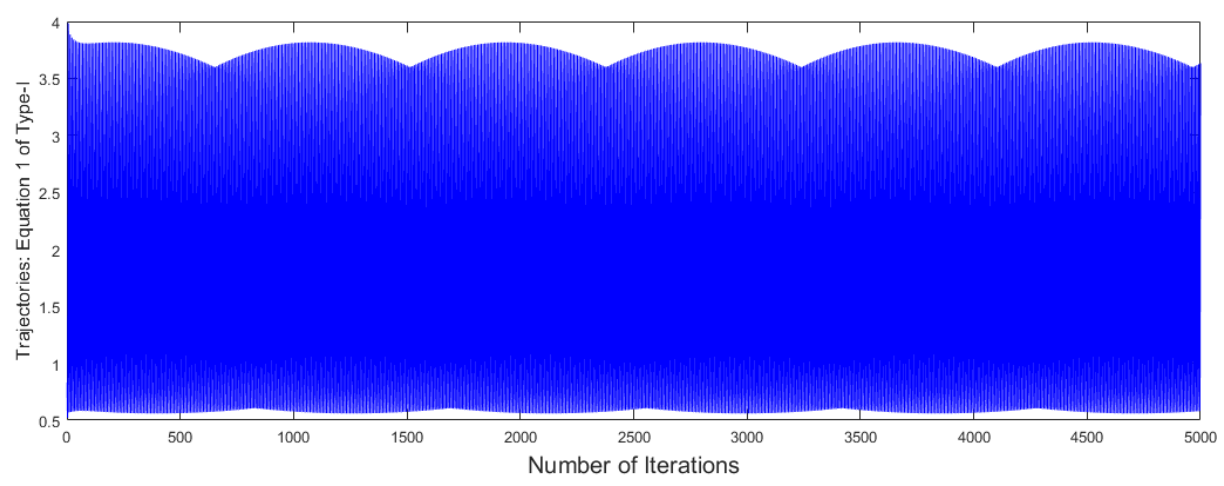

Figure 16: Quasi-periodic solution of the RDE 1.

In the Example 3.3 and 3.4, the trajectories in the Fig.15 and Fig.16 are turning out to be a self-similar fractal [23]. The fractal dimensions of the trajectories of the figures are enumerated through the Benoit software (for quick reference, the snapshot of the calculation is given in the Fig.17) and found to be 1.862 and 1.871 respectively 24 .

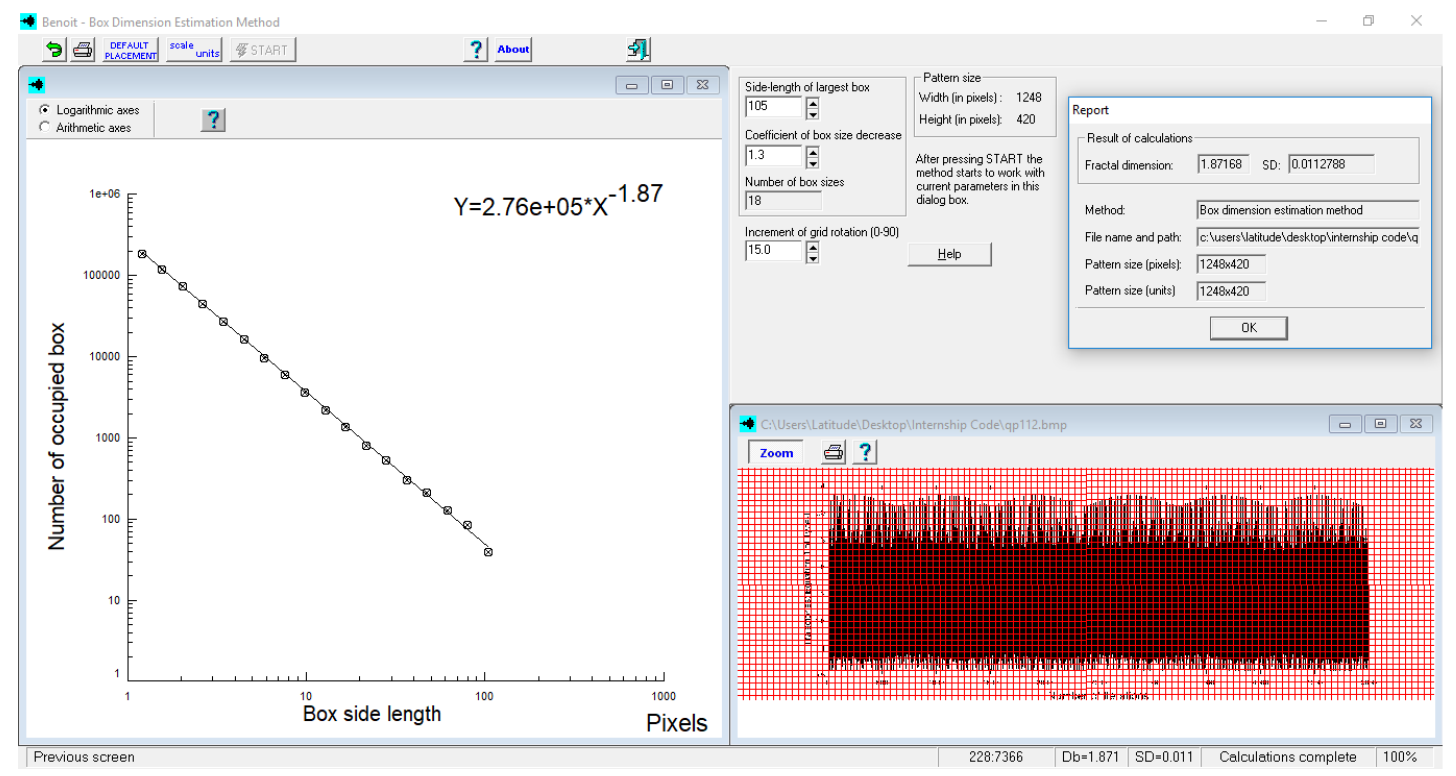

Figure 17: Fractal dimension calculation through Benoit of the trajectory as shown in Fig.16

The RDE 3 possesses period three solutions $0,0, \frac{1}{c}, 0,0, \frac{1}{c}, \ldots$. which is evident from the equation whenever the initials are taken near the period three cycle $0,0, \frac{1}{c}$. Some examples are given as follows:

Example 3.5. Consider $a=0.8454, b=0.8789$ and $c=0.7462$ in the $R D E 3$ we get period three solutions as $0,0,1.3402,0,0,1.3402, \ldots \ldots$ which is shown in the following Fig. 18. 


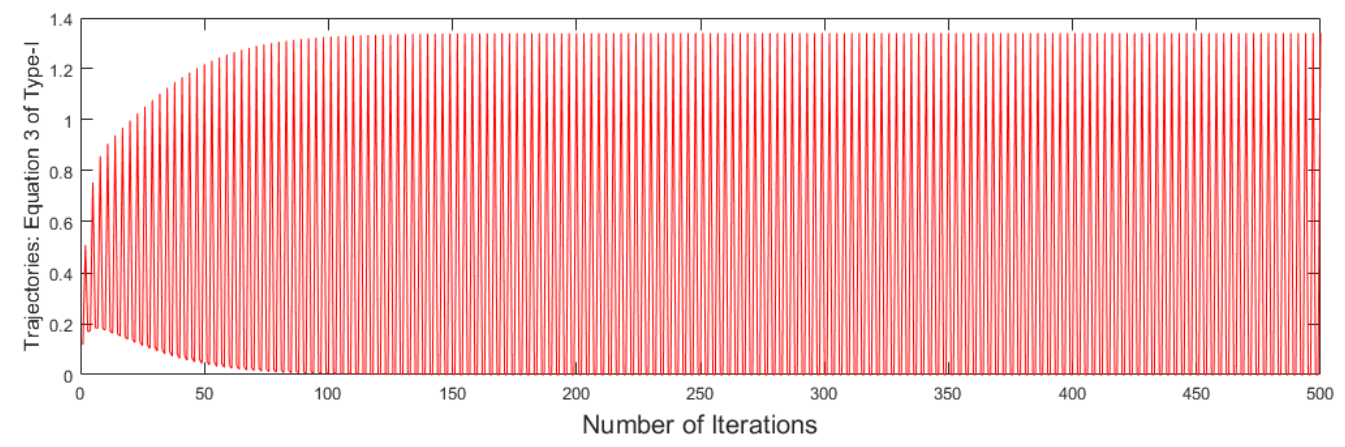

Figure 18: Period three solutions $0,0,1.3402,0,0,1.3402, \ldots \ldots$ of the RDE 3 .

It is noted that the initial values $x_{-2}, x_{-1}$ and $x_{0}$ are taken from the $\epsilon$-neighbourhood of $0,0, \frac{1}{c}$ respectively.

Example 3.6. Consider $a=0.7242, b=0.5809$ and $c=0.5403$ in the $R D E 3$ we get period three solutions as $0,0,1.851,0,0,1.851, \ldots \ldots$ which is shown in the following Fig. 19.

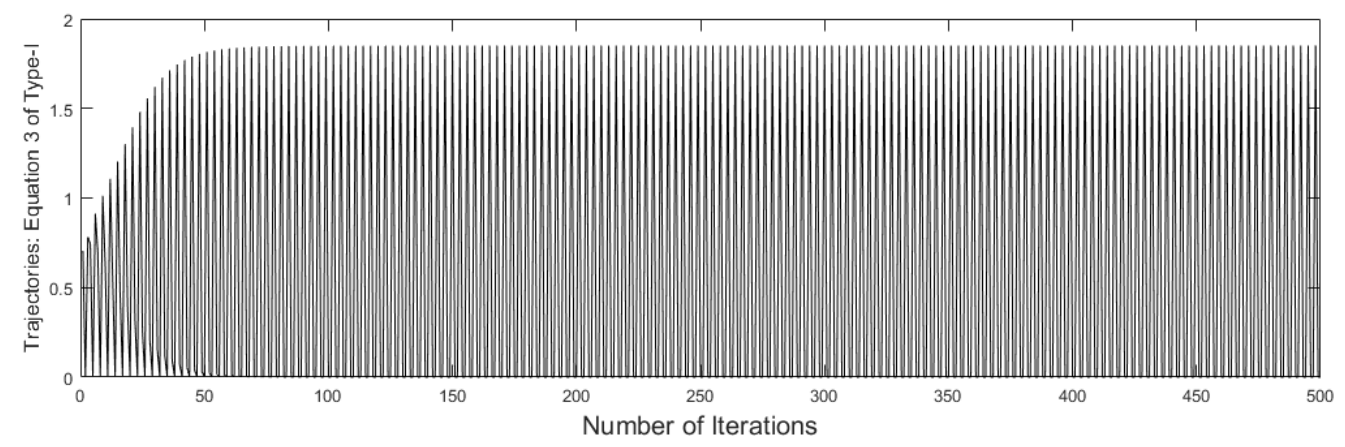

Figure 19: Period three solutions $0,0,1.851,0,0,1.851, \ldots \ldots$ of the RDE 3.

As usual the initial values $x_{-2}, x_{-1}$ and $x_{0}$ are taken from the $\epsilon$-neighbourhood of $0,0, \frac{1}{c}$ respectively.

Remark 3.1. As observed in the Examples 3.5 and 3.6, it is observed that the period three solutions $0,0, \frac{1}{c}, 0,0, \frac{1}{c}, \ldots, \ldots$ is locally asymptotically stable.

We also encounter some quasi-periodic (near-periodic) with high quasi-period (near-period) solutions of the RDE 3 of Type-I as shown in the following two examples.

Example 3.7. Consider $a=0.3417, b=0.5487$ and $c=0.5605$ with initial values taken from the $\epsilon$-nbd of the zero, the trajectory possesses to a very high quasi periodic solution as shown in Fig.20. 


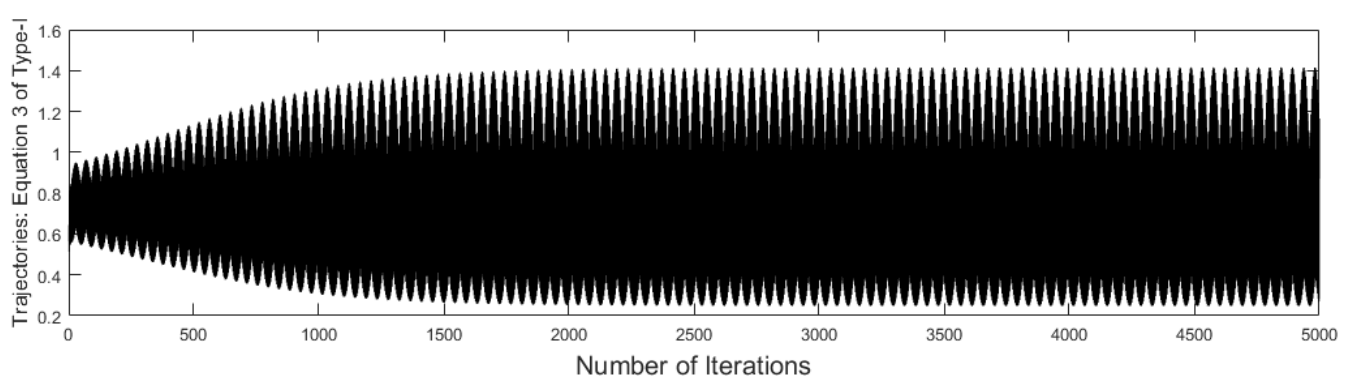

Figure 20: Quasi-periodic solution of the RDE 3.

Example 3.8. Consider $a=0.0013, b=0.9813$ and $c=0.5702$ with initial values taken from the $\epsilon$ - $n b d$ of the zero, the trajectory possesses to a very high quasi periodic solution as shown in Fig.21.

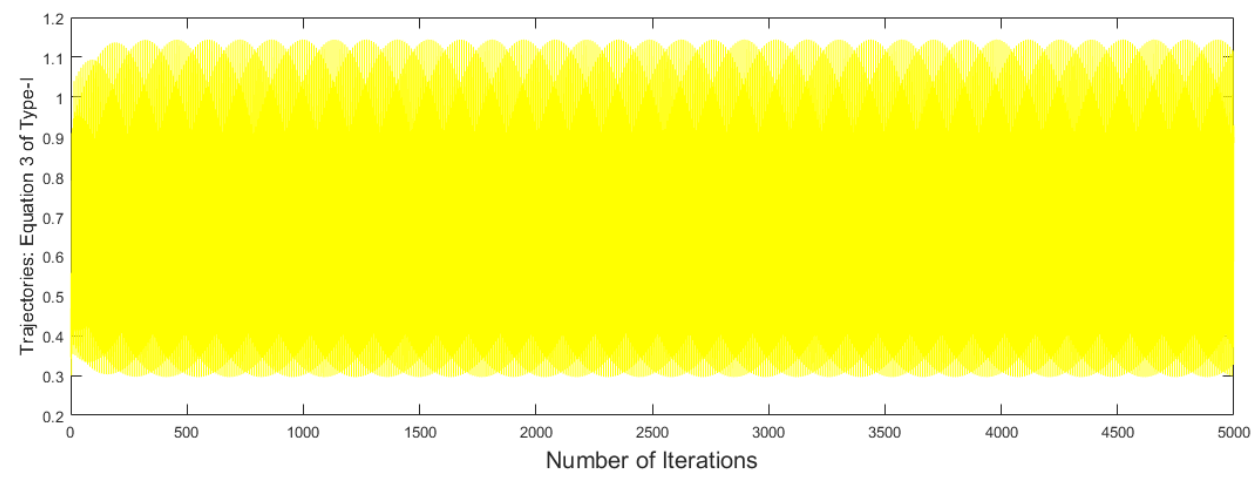

Figure 21: Quasi-periodic solution of the RDE 3.

In the Example 3.7 and 3.8, the trajectories in the Fig.20 and Fig.21 are turning out to be a self-similar fractal. The fractal dimensions of the trajectories of the figures are enumerated through the Benoit software and found to be 1.713 and 1.817 respectively .

\subsection{Periodic Solutions of the RDEs of Type-II}

We have seen the prime period two solutions of the RDEs 4 and 6 of Type-II exist whenever $b=a+c$. This is illustrated here through the following examples as shown in Table 4.

\begin{tabular}{|c|c|l|}
\hline Parameters $b=a+c$ & \multicolumn{1}{|c|}{$\phi, \psi$} & Remarks \\
\hline $\begin{array}{c}\mathrm{a}=0.3689, \mathrm{c}=0.4607- \\
\text { of the RDE } 4\end{array}$ & $\phi=1.48, \psi=1.88$ & as shown in Fig. 22 (Row-1, Col-1) \\
\hline $\begin{array}{c}\mathrm{a}=0.6448, \mathrm{c}=0.3763- \\
\text { of the RDE } 4\end{array}$ & $\phi=3.1278, \psi=1.5159$ & as shown in Fig. 22 (Row-1, Col-2) \\
\hline $\begin{array}{c}\mathrm{a}=0.6596, \mathrm{c}=0.5186- \\
\text { of the RDE } 6\end{array}$ & $\phi=2.2753, \psi=2.4435$ & as shown in Fig. 22 (Row-2, Col-1) \\
\hline $\begin{array}{c}\mathrm{a}=0.1734, \mathrm{c}=0.3909- \\
\text { of the RDE } 6\end{array}$ & $\phi=0.6761, \psi=3.4136$ & as shown in Fig. 22 (Row-2, Col-2) \\
\hline
\end{tabular}

Table 4: Parameters with corresponding prime period two solutions when $b=a+c$ of the RDEs 4 and 6 

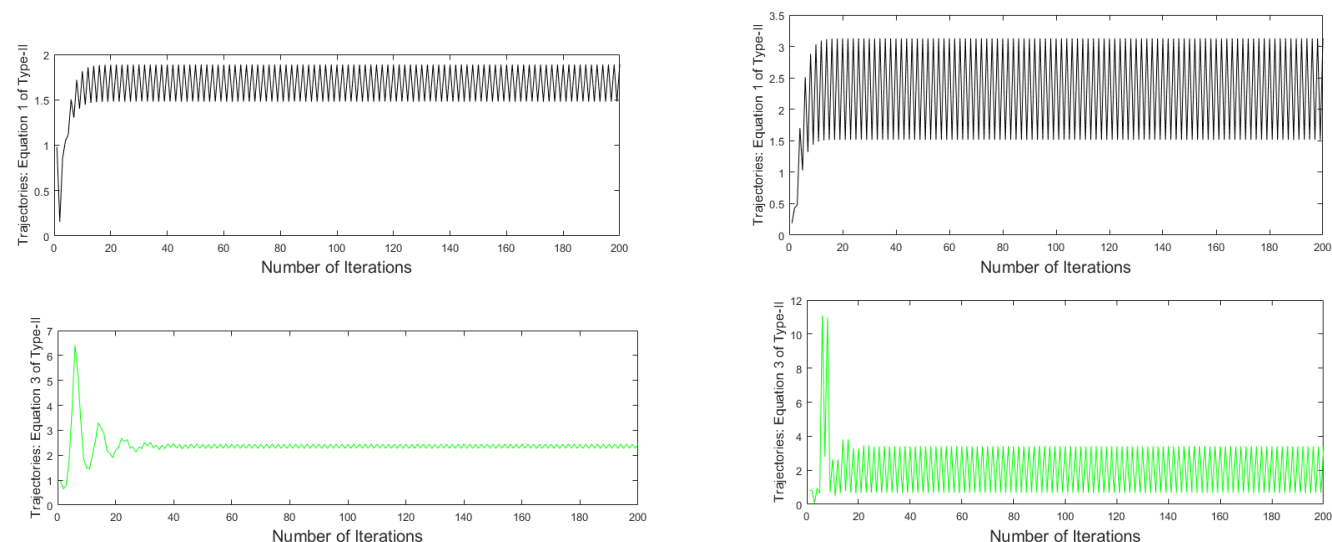

Figure 22: Prime period two solutions of the RDEs 4 and 6

The RDE 5 of Type-II possesses prime period two solutions provided $\phi+\psi=b-a-c$. For certain ranges of parameters $a, b, c$, prime period solutions $\phi, \psi, \phi, \psi, \ldots$ occur.
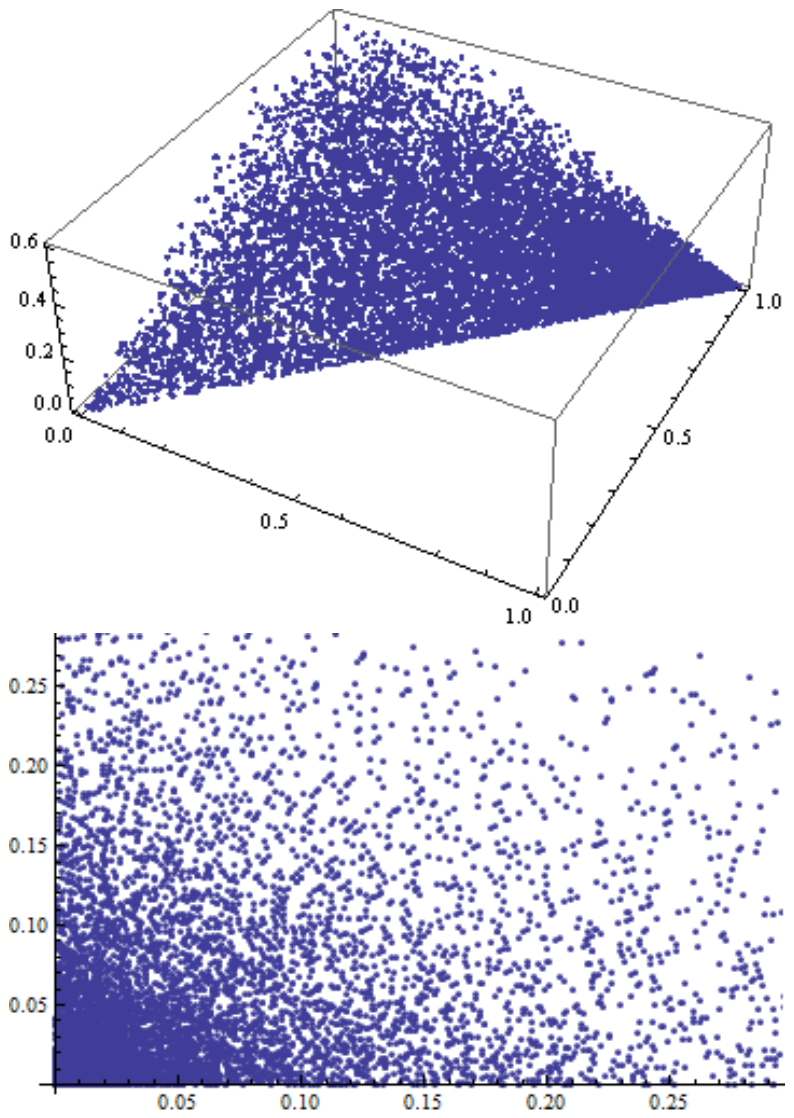

Figure 23: Parameters $(a, b, c)$ in 3D with Prime period two solutions in 2D of the RDE 5 of Type-II 
Here We have omitted examples in this case. Rather we give a set of parameters such that the prime period solutions happen. Also such parameters as points $(a, b, c)$ in three dimension are plotted with in two dimension the prime period two solutions are plotted in the Fig. 22.

\section{A Comparative View of Dynamics of RDEs}

In this section, we shall describe a comparative view through examples of the dynamical behavior of the rational difference equations of both the Type-I and Type-II [22].

Example 4.1. Let $a=0.7094, b=0.7547$ and $c=0.2760$ with initial conditions taken from the $\epsilon-n b d$ of zero, the trajectories for the RDEs 1 and 3 converge to $0.5747\left(\frac{1}{a+b+c}\right)$ whereas the RDEs 4, 5 and 6 converge to $1.7401(a+b+c)$. The RDE 2 of Type-I possesses to prime period two solutions $0,1.3251,0,1.3251, \ldots$ The trajectories are shown in the following Fig. 24.
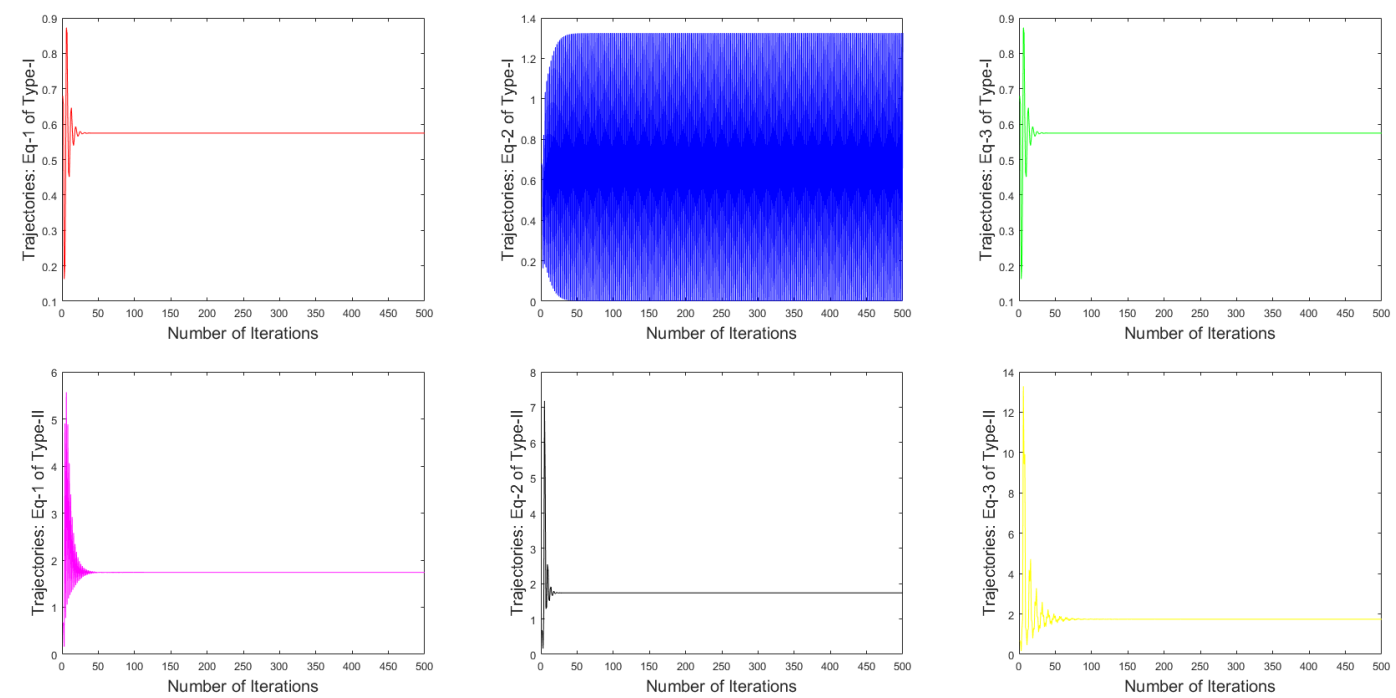

Figure 24: Dynamics of the RDEs when $a=0.7094, b=0.7547$ and $c=0.2760$

Example 4.2. Let $a=0.0759, b=0.0540$ and $c=0.5308$ with initial conditions taken from the $\epsilon-n b d$ of zero, the RDEs 1 and 3 possess to the same period eight solution 11.6963, 6.5060, 1.1118, 0.1673, 0.0475, 0.0787,0.8086, 8.9038 whereas the RDE 2 exhibits prime period two solutions $0,18.5185,0,18.5185, \ldots$ The RDEs 4, 5 lead to quasi-periodic solutions (fractal dimensions are 1.794 and 1.6987 respectively) with high period whereas the RDE 6 converges to 0.6607 [?]. The trajectories are shown in the Fig. 25. 

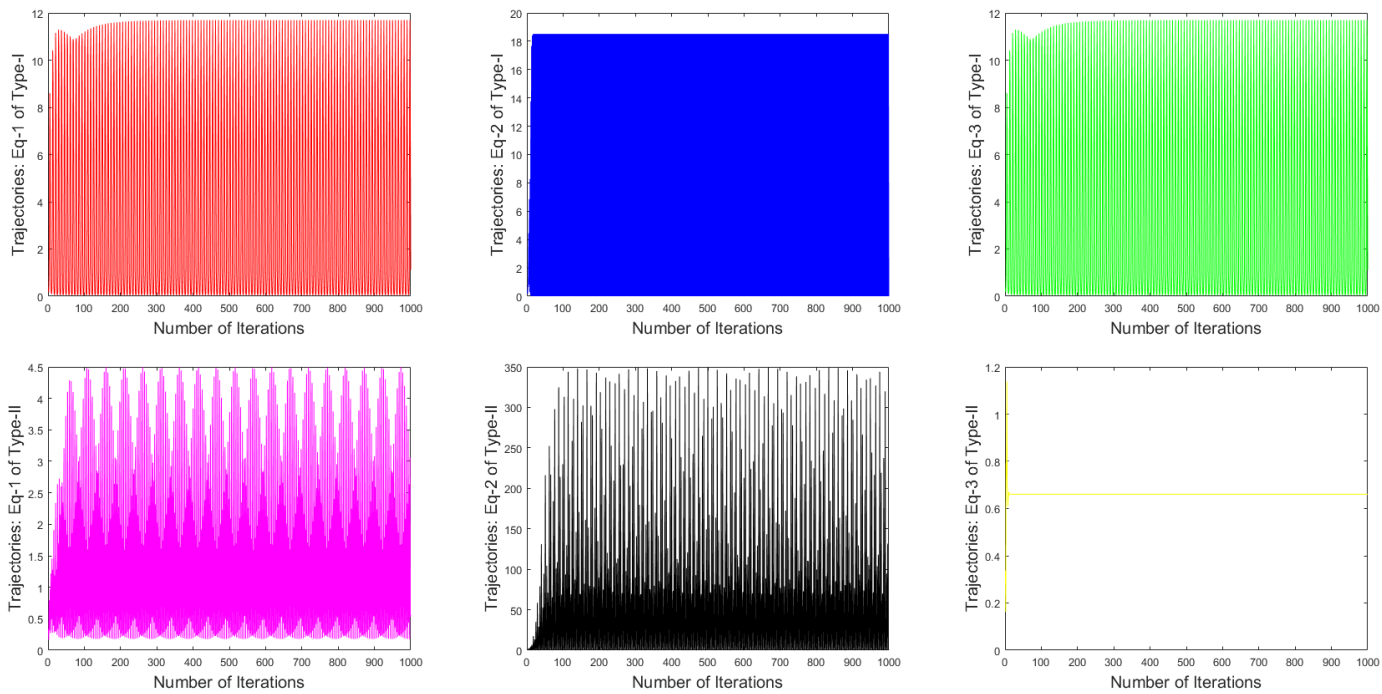

Figure 25: Dynamics of the RDEs when $a=0.0759, b=0.0540$ and $c=0.5308$

Example 4.3. Let $a=0.3112, b=0.5285$ and $c=0.1656$ with initial conditions taken from the $\epsilon-n b d$ of zero, all the RDEs of Type-I possess to convergence and converge to 0.9946 whereas the RDE 4 and 6 of Type-II blows up to unbounded solutions. The RDE 5 possesses to convergence and converges to 1.0054. The trajectories are shown in the following Fig. 26.
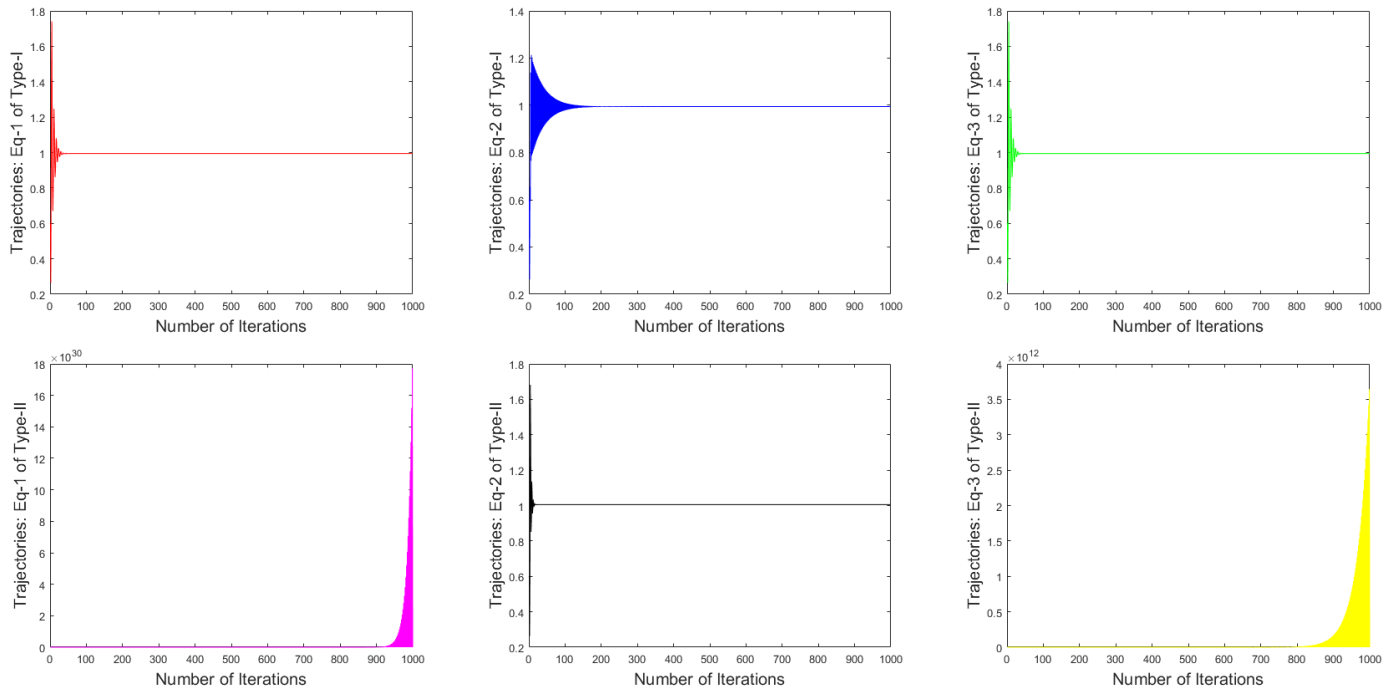

Figure 26: Dynamics of the RDEs when $a=0.0759, b=0.0540$ and $c=0.5308$

Example 4.4. Let $a=0.2630, b=0.9171(a+c)$ and $c=0.6541$ with initial conditions taken from the $\epsilon-n b d$ of zero, then the RDEs 1 and 3 of Type-I possess to convergence and converge to 0.5452 whereas the RDE 2 of Type-I leads to a prime period two solutions where $\phi=0.6394$ and $\psi=4510$. The RDE 4 possesses to prime period two solution $13.5287,0.9837,13.5287,0.9837, \ldots$ and the 6 leads 
to the prime period solution $1.3642,2.7980,1.3642,2.7980, \ldots$ whereas the RDE 5 converges to 1.8341 . The trajectories are shown in the following Fig. 27.
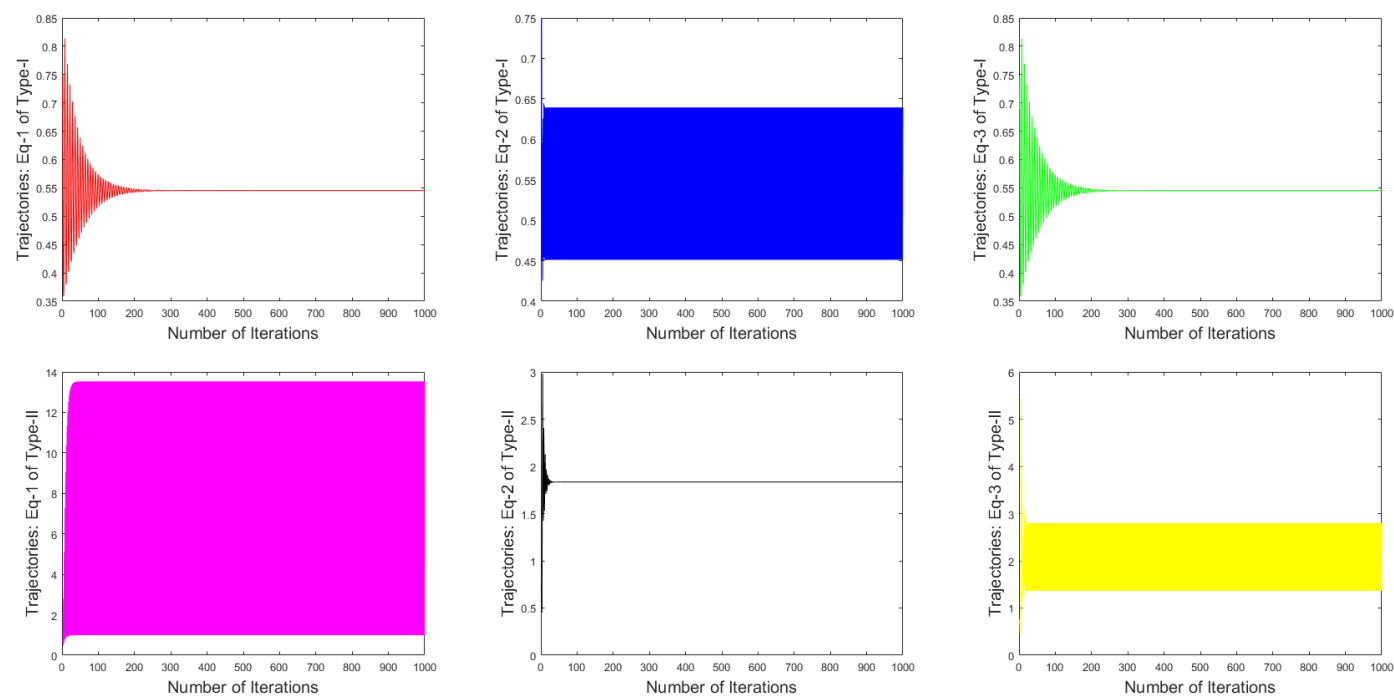

Figure 27: Dynamics of the RDEs when $a=0.2630, b=0.9171(a+c)$ and $c=0.6541$

Based on the examples cited the following remarks are drawn.

Remark 4.1. Every solutions of the RDEs of Type-I are bounded. Either solutions are convergent or possess to periodic (quasi-periodic) solutions. The solutions of the RDEs of Type-II might go unbounded as shown in above Example 4.3.

Remark 4.2. Every solutions of the all the RDEs either convergent or possesses to periodic (quasiperiodic) solutions whenever $b=a+c$.

\section{Conclusions \& Future Endeavours}

This present article describes the dynamical behavior of the class of rational difference equations of two types. They exhibit various dynamics with variations. This is possibly mathematical unity in diversity. For certain parameters the RDEs agree with local asymptotic stability and there are parameters under which each difference equations are self-sensitive. It is noted that no chaotic trajectory is seen in these rational difference equations for positive real parameters and initial conditions. We expect various exotic dynamical behaviors such as (fractal, chaotic, high periods etc) if parameters and initial values can be made over entire real line. There are certainly many other aspect of the dynamical behavior which are due to be seen in near future. There several other class of rational difference equations could be studied computationally in order to apprehend their comparative dynamics.

\section{Acknowledgement}

The first author is grateful to the Pingla Thana Mahavidyalaya for the support by providing the Summer-Interns (2019) in carrying out the present research. The other intern authors also express their gratitude for the accomplishment during the joyful experiences of the Summer Internship 2019 at the Pingla Thana Mahavidyalaya. 


\section{References}

[1] R. Agarwal, Difference Equations and Inequalities, Theory, Methods and Applications, Marcel Dekker Inc., New York 1992.

[2] S. Elaydi, An Introduction to Difference Equations, 3rd ed., Springer-Verlag, New York, 2005.

[3] V. Hutson and K. Schmidtt, Persistence and the dynamics of biological systems, Math. Biosc., 11(1992), 1-71.

[4] H. Sedaghat, Nonlinear Difference Equations, Theory and Applications to Social Science Models, Kluwer Academic Publishers, Dordrecht, 2003.

[5] E.C. Pielou, An Introduction to Mathematical Ecology, Wiley-Interscience, New York, 1969.

[6] G. Ladas, G. Tzanetopoulos, and A. Tovbis, On May's host parasitoid model, J. Difference Equ. Appl., 2(1996), 195-204.

[7] R. Abo-Zeid, (2015) On the oscillation of a third order rational difference equation, Journal of the Egyptian Mathematical Society, 23, 62-66.

[8] Sk. S. Hassan, E. Chatterjee, (2015) Dynamics of the equation $z_{n+1}=\frac{\alpha+\beta z_{n}}{A+z_{n-1}}$ in the Complex Plane, Cogent Mathematics, Taylor and Francis 2, $1-12$.

[9] Esha Chatterjee, Sk. S. Hassan, (2018) On the asymptotic charecter of a generalized rational difference equation, Discrete \& Continous Dynamical Systems-A, 38(4), 1707-1718.

[10] H.L. Smith, Monotone dynamical systems. An introduction to the theory of competitive and cooperative systems, Math. Surveys Monogr., Vol. 41 , Amer. Math. Soc., Providence, RI, 1995.

[11] Elias Camouzis and Gerasimos Ladas, (2009) Dynamics of Third-Order Rational Difference Equations with Open Problems and Conjectures, Taylor $\&$ Francis Group, Chapman 83 Hall/CRC.

[12] E. Camouzis, E. Chatterjee, G. Ladas and E. P. Quinn, (2004) On Third Order Rational Difference Equations Open Problems and Conjectures, Journal of Difference Equations and Applications, 10, $1119-1127$.

[13] E. Camouzis and G. Ladas, On third-order rational difference equations, Part 1 and Part 2, J. Difference Equ. Appl., (2007)

[14] W.G. Kelley and A.C. Peterson, Difference Equations, Academic Press, New York, 1991.

[15] E. Magnucka-Blandzi and J. Popenda, On the asymptotic behavior of a rational system of difference equations, J. Difference Equ. Appl., 5(1999), 271-286.

[16] S. Elaydi and R.J. Sacker, Global stability of periodic orbits of nonautonomous difference equations in population biology and the Cushing-Henson conjectures, Proc. 8th Int. Conf. Difference Equat. Appl., Brno, Czech Republic, 2003.

[17] S. Elaydi and R.J. Sacker, Global stability of periodic orbits of nonautonomous difference equations and population biology, J. Differential Equ., 208(2004), 258-273.

[18] G. Ladas, Open problems and conjectures, J. Difference Equ. Appl., 4(1998), 93-94.

[19] R. Abu-Saris and Kh. Al-Dosary, Invariants: A functional-equation approach, J. Difference Equ. Appl., 10(2004), 1037-1040. 
[20] E.A. Grove and G. Ladas, Periodicities in Nonlinear Difference Equations, Chapman \& Hall/CRC Press, 2005.

[21] L. Berg, Nonlinear difference equations with periodic solutions, Rostock. Math. Colloq., 61(2006), 12-19.

[22] A.M. Amleh, E. Camouzis, and G. Ladas, On the boundedness character of rational equations, Part 2, J. Difference Equ. Appl., 12 (2006), 637-650.

[23] H.D. Peitgen and D. Sanpe, The Science of Fractal Images, Springer-Verlag, New York, 1988.

[24] X. C. Jin, Jayasooriah, A practical method for estimating fractal dimension, Pattern Recognition Letters, 16(5), (1995), 457-464. 